\title{
Salmonellose de la poule à Salmonella pikine
}

\author{
par F. SAGNA avec la collaboration de M. NIANG et M. A. THIOUNE
}

\begin{abstract}
RÉSUMÉ
Solmanelia pikine a été isolée pour la première fois à Dakar par S. et L. LE MINOR, P. KIRSCHE, R. BAYLET et J. SAMAILLE en 1958 à partır d'un ganglion mésentérique de porc apparemment normal.

Cette souche est de nouveau isolée en mars 1968, à Dakar, au Laboratore national de l'Elevage et de Recherches vétérinaires, à partir de poulets malades provenant d'un élevage familıal de la zone suburbaıne de Dakar. Le pouvoir pathogène de ce sérotype vis-à-vis des volailles est ainsi confirmé.
\end{abstract}

Salmonella pıkine a été isolée pour la première fois à Dakar par S. et L. LE MINOR, P. KIRSCHE, R. BAYLET et J. SAMAILLE en 1958, par «ensemencement de broyat de ganglions mésentériques de porc apparemment normal sur milieu 5 S, après enrichissement sur milieu de MÜLLERKAUFFMANN》(1). II s'agissait alors d'une enquête par sondage visant à recenser les différents sérotypes de Salmonella hébergés par cet animal à l'occasion de prélèvements systématiques à l'abattoir de Dakar. Mais aucune relation ne pouvalt être faite alors entre l'isolement de ce nouveau sérotype chez un porc et l'existence d'un pouvoir pathogène éventuel de cette souche vis-à-vis de cette espèce domestique.

En mars 1968, cette souche est isolée de nouveau, toujours à Dakar, mais chez des poulets malades, dans les circonstances suivantes:

Le 23 mars 1968, M. G... qui exploite à Dakar au Point $E$ (zone A) un élevage avicole de type familial se présente à la section de pathologie aviaire du Laboratoire national de l'Elevage et de Recherches vétérinaires du Sénégal avec cinq poulets malades.

Un examen clinique révèle des sujets adynamiques, très amaigris et présentant de la diarrhée.

Une maladie parasitaire est tout d'abord suspectée. Le poulet le plus affaibli du lot est sacri- fié et autopsié. Aucune lésıon organique n'est visible à l'exception de l'entérite. Des prélèvements obtenus par raclage de la muqueuse digestive, des excréments, sont examinés au microscope, aınsı que les excréments de tous les olseaux maiades. Tous ces examens se révélant négatifs, l'idée de parasitose est abandonnée, et l'hypothèse d'une diarrhée infectieuse d'origine microbienne est alors émise.

La moelle d'un os long et le sang du cœur du sujet sacrifié sont ensemencés en bouillonsérum et en bouillon du type MULLER-KAUFFMANN. Après repiquage sur milieux solides (gélose nutritive et gélose $S$ S), un germe est obtenu en culture pure. Il possède les caractères suivants :

- bactérie gram négatıf, très mobıle, glucose + (gaz), lactose - $\mathrm{SH}_{2}+$, indol et uréase -, LDC + en milieux glucose-lactose$\mathrm{SH} 2$ et de Carlquist : $\beta$-galactosidase - avec le test à l'ONPG.

Ces caractères faisant penser à une salmonelle, diverses agglutinations rapides sur lames sont pratiquées et donnent les résultats suivanłs :

- sérums $\mathrm{O}$ mélanges : $\mathrm{OMB}+$

- sérums anti-O : $6-8+$

- sérums anti-H $: r+$ en phase 1 (phase 2 ?). 
Les caractères biochimiques complémentaires de cette saimonelle sont les suivants :

- citrate +, RM +, VP — ; mannitol, sorbitol, galactose, lévulose, maltose, mannose, arabinose, raffinose : + en $24 \mathrm{~h}$; rhamnose +- ; l'adonitol, l'inositol, le dulcitol, le saccharose et l'inuline ne sont pas fermentés.

En l'absence de la gamme complète des sérums agglutinants, cette souche de Salmonelle est lyophilisée et adressée au Centre national français des Salmonelles, à l'Institut Pasteur de Paris, qui confirme qu'il s'agit bien d'une Salmonelle, «Salmonella pikme,» dont le sérotype complet est (8)20:r:Z 6 .

Ainsi le pouvoir pathogène de Salmonella pikine vis-à-vis de la poule est démontré.

Le porc, coprophage, peut s'infecter et héberger le germe sans trouble apparent, aınsı que l'ont démontré LE MINOR et Coll.

En conclusion, ce nouveau sérotype vient s'ajouter à la liste déjà nombreuse des Salmo- nelles reconnues pathogènes pour la volaille, capable de déclencher des troubles digestifs graves risquant de compromettre la marche normale des élevages.

Le pouvoir pathogène de ce sérotype vis-à-vis du porc, hôte démontré, n'est pas à écarter.

A la lumière de ces faits, il importe de rappeler que la lutte contre les salmonelloses aviaires et porcines exige l'application stricte des mesures d'hyglène générale de l'élevage et la séparation des espèces sensibles.

Institut d'Elevoge ef de Médecine vétérinaire des Pays tropicaux Laboratorre national de l'Elevage ef de Recherches vétérmoires Dakar.

Qu'il nous soit permis de remercier ici notre Maître, le Professeur LE MINOR, qui a bien voulu se charger de la détermination sérologique complète de cette souche et nous a adressé divers documents sur les plus récents schémas de classification des Salmonella.

\section{SUMMARY}

Salmonallosis of hen with Salmonella Pikine

Salmonella pikine was isolated for the first time in Dakar by S. and L. LEMINOR, P. KIRSCHE, R. BAYLET and J. SAMAILLE in 1958 from a mesenteric lymph node of an apparently healthy swine.

This strain is agan isolated in march 1968, in the National Animal Husbandry and Veterinary Research Laboratory of Dakar, from ill chıcks of a family poultry-yard in suburban zone of Dakar. So the pathogenic power of this serotype in regard to the fowls is confirmed.

\section{RESUMEN}

Salmonelosis con Salmonella pikine de la gallina

S. y L. LE MINOR, P. KIRSCHE, R. BAYLET y J. SAMAiLLE aislaron Solmonella pikine por vez primera en 1958, en Dakar, a partır de ganglios mesenté ricos de un cerdo aparentemente normal.

De nuevo se ass/a dicha cepa en marzo de 1968 , en el laboratorio nacional de la crianza y de investıgaciónes veterinarias de Dakar, a partir de pollos enfermos proviniendo de una crıanza familial de la zona suburbana de Dakar. Asi se confirma el poder patógeno de este serotipo para con las aves de corral.

\section{BIBLIOG RAPHIE}

1. LE MINOR (S. et L.), KIRSCHE (P.), BAYLET (R.) et SAMAILLE (J.). -. Un nouveau sérotype de Salmonella : Salmonella pikine (8) 20:r: Z 6. - Annales de l'Institut Pasteur, $1958,95,218-219$. 